\title{
Esfuerzo-recompensa, demanda-control y satisfacción con la vida: un estudio con docentes de educación primaria
}

\author{
Effort-Reward, Demand-Control and Satisfaction with Life: A Study with \\ Elementary Teachers
}

Esforço-recompensa, demanda-controle e satisfação com a vida: um estudo com professores do ensino fundamental

José Ángel Vera Noriega

Universidad de Sonora, México

jose.vera@unison.mx

http://orcid.org/0000-0003-2764-4431

Jazmín Valdez Tam

Centro de Estudios Educativos y Sindicales de la Sección 54 del Sindicato Nacional de Trabajadores de la Educación, México profesora.jazmin@hotmail.com https://orcid.org/0000-0003-0206-8343

Erika Selene Contreras Grijalva Centro de Estudios Educativos y Sindicales de la Sección 54 del Sindicato Nacional de Trabajadores de la Educación, México eriikacont89@gmail.com https://orcid.org/0000-0002-6937-6466

Silvia Selene Castillo Velasco Centro de Estudios Educativos y Sindicales de la Sección 54 del Sindicato Nacional de Trabajadores de la Educación, México sylviacastillo93@hotmail.com https://orcid.org/0000-0001-8367-4796 


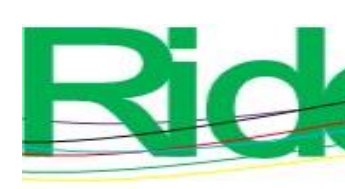

Revista Iberoamericana para la

Investigación y el Desarrollo Educativo

ISSN 2007-7467

\section{Resumen}

En nuestro país no existe suficiente información sobre los factores psicosociales que afectan la salud mental de los docentes, lo cual es un obstáculo para la implementación de políticas que atiendan esta problemática. Los factores psicosociales de riesgo en el trabajo docente son poco estudiados y se requiere vincular los factores personales de satisfacción con la vida y los de riesgo laboral para dar cuenta sobre la forma en la cual las percepciones personales pueden amortiguar los impactos de los riesgos laborales. Por ello, el objetivo de esta investigación fue estudiar con docentes de primaria la comparación entre las variables satisfacción con la vida, percepción de esfuerzo-recompensa y demanda-control con los factores sexo, edad, antigüedad en la docencia, antigüedad en el centro de trabajo y atención a alumnos con necesidades educativas especiales. Se utilizó un diseño retrospectivo de alcance descriptivo y comparativo. La muestra de estudio estuvo compuesta por 185 docentes de educación primaria pertenecientes al municipio de Cajeme, Sonora. Para el levantamiento de datos se aplicó la escala de satisfacción con la vida de Diener, el cuestionario de estrés laboral (desbalance esfuerzo-recompensa) en su versión venezolana validado por Díaz y Feldman y el cuestionario de salud general de Goldberg. Se obtuvieron los deciles para cada una de las variables. La variable demanda-control obtuvo en el primer decil el valor de 3.00, lo cual coloca a 22 personas (11.9\%) debajo de este valor, lo cual significa que se encuentran en riesgo de un problema de salud mental. Por otra parte, en la variable percepción esfuerzo-recompensa 21 individuos (11.4\%) presentaron niveles por debajo del primer decil con un valor de 2.68 y, por tanto, desequilibrado. Por último, el valor del primer decil fue de 3.40 para 22 personas $(11.9 \%)$, lo que representa los niveles más bajos en la escala de satisfacción con la vida. Las comparaciones mostraron que el número de alumnos con necesidades de educación especial atendidos influye en las variables satisfacción con la vida, percepción esfuerzorecompensa y demanda-control, ya que al aumentar el número de alumnos atendidos disminuyó la media en las tres variables. Por otro lado, el tiempo es un factor significativo en la variable esfuerzo-recompensa, ya que al aumentar la edad, los años de servicio y la antigüedad en el centro de trabajo, la media en esta variable fue disminuyendo. Existe una relación de las variables satisfacción con la vida y esfuerzo-recompensa con los factores de temporalidad (edad, años de experiencia en la docencia y años de experiencia en la escuela), aunque se considera necesario indagar en ese aspecto. Resulta importante destacar que avanzar en el estudio de los factores psicosociales que pueden afectar la satisfacción y salud laboral del magisterio es fundamental para la toma de decisiones de políticas para el mejoramiento de la educación en México. Es necesario 


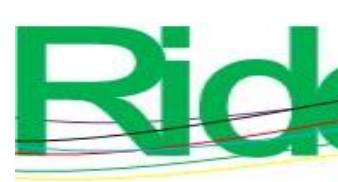

Revista Iberoamericana para la Investigación y el Desarrollo Educativo ISSN $2007-7467$

ampliar este tipo de investigaciones incluyendo maestros de diferentes niveles y contextos, de escuelas públicas y privadas, con el fin de obtener información más precisa.

Palabras clave: condiciones de empleo del docente, docente de escuela primaria, factores psicológicos, satisfacción laboral.

\section{Abstract}

In our country there doesn't exist enough information about psychosocial factors that affect teacher's mental health, which it's an obstacle for the policies implementation that fight against this issue. Psychosocial factors of labor risk in teaching work are barely studied and is required to link personal factors of life satisfaction and those of labor risk to realize about the way personal perceptions can cushion the impacts of labor risks. The objective of this investigation was to study with elementary school teachers, the comparison between life satisfaction, effort-reward perception and demand- control with the following factors: sex, age, antiquity in teaching, time in the workplace and attention to students with special educational needs. A retrospective design with a descriptive and comparative scope was used. The studied population consisted of 185 elementary school teachers of Cajeme, Sonora. In order to collect the information, the Diener Life Satisfaction Scale, the Labor Stress Questionnaire (Effort-Reward Imbalance) in its Venezuelan version, validated by Díaz and Feldman, and the General Health Questionnaire of Goldberg were applied. Deciles were obtained for every one of the variables. The demand-control variable obtained the value of 3.00 in the first decile, which places 22 people (11.9\%) below this value, this means they are at risk of a mental health problem. On the other hand, on the perception of effhort-reward variable 21 individuals (11.4\%) presented levels below the first decil with a 2.68 value and, so, unbalanced. By last, the firs decil value was 3.40 for 22 people (11.9\%) which represent the lowest levels on the satisfaction with life scale. The comparisons showed that the number of students with special educational needs attended influenced the variables of satisfaction with life, perception of effort-reward and demand-control, since as the number of attended students increased, the average in the three variables decreased. On the other hand, time is a significant factor in the effort-reward variable since with increasing age, years of service and antiquity in the workplace the average in this variable decreased. There is a relation between the variables of satisfaction with life, effortreward and demand-control with temporality factors (age, years of service and antiquity in the workplace), however, it is considered necessary to inquire into that aspect. It is important to highlight that progress in the study of psychosocial factors that can affect job satisfaction and 


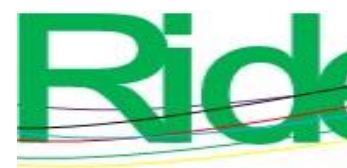

Revista Iberoamericana para la
Investigación y el Desarrollo Educativo
ISSN $2007-7467$

health in teachers is essential for decision making around policies for education improvement in Mexico. It is necessary to expand this type of research including teachers of different levels and contexts, from public and private schools, in order to obtain more accurate information.

Keywords: teacher employment conditions, elementary school teacher, psychosocial factors, work satisfaction.

\section{Resumo}

Em nosso país, não há informações suficientes sobre os fatores psicossociais que afetam a saúde mental dos professores, o que dificulta a implementação de políticas que abordem essa problemática. Os fatores de risco psicossocial no trabalho docente são pouco estudados e é necessário relacionar os fatores pessoais de satisfação com a vida e os de risco ocupacional para explicar a forma como as percepções pessoais podem amortecer os impactos dos riscos ocupacionais. Portanto, o objetivo desta pesquisa foi estudar com professores do ensino fundamental a comparação entre as variáveis satisfação com a vida, percepção de esforçorecompensa e demanda-controle com os fatores sexo, idade, tempo de docência, tempo de internação. centro de trabalho e atendimento a alunos com necessidades educacionais especiais. Foi utilizado um desenho retrospectivo com escopo descritivo e comparativo. A amostra do estudo foi composta por 185 professores do ensino fundamental do município de Cajeme, Sonora. Para a coleta de dados, foram aplicados a escala Diener de satisfação com a vida, o questionário de estresse no trabalho (desequilíbrio esforço-recompensa) em sua versão venezuelana, validado por Díaz e Feldman, e o questionário geral de saúde de Goldberg. Decis foram obtidos para cada uma das variáveis. A variável demanda-controle obteve no primeiro decil o valor de 3,00, o que coloca 22 pessoas $(11,9 \%)$ abaixo desse valor, o que significa que estão em risco de desenvolver um problema de saúde mental. Por outro lado, na variável percepção esforço-recompensa, 21 indivíduos $(11,4 \%)$ apresentaram níveis abaixo do primeiro decil com valor de 2,68 e, portanto, desequilibrados. Por fim, o valor do primeiro decil foi de 3,40 para 22 pessoas $(11,9 \%)$, o que representa os níveis mais baixos na escala de satisfação com a vida. As comparações mostraram que o número de alunos atendidos com necessidades educacionais especiais influencia as variáveis satisfação com a vida, percepção esforço-recompensa e demanda-controle, pois à medida que o número de alunos atendidos aumenta, a média nas três variáveis diminui. Por outro lado, o tempo é um fator significativo na variável esforço-recompensa, visto que com o aumento da idade, anos de serviço e tempo de serviço no local de trabalho, a média dessa variável diminuiu. Existe relação 


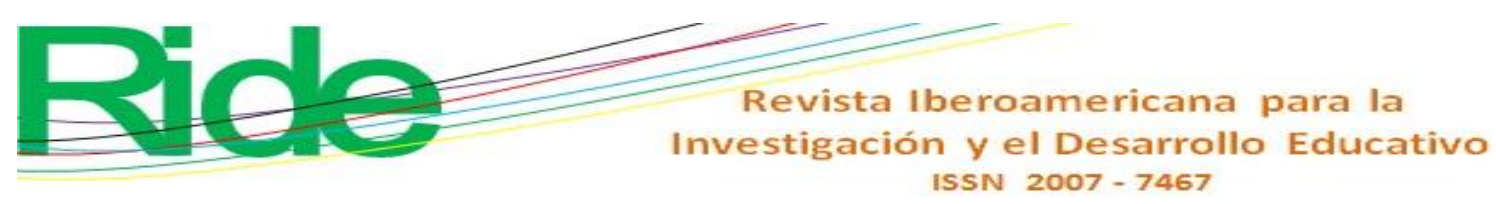

entre as variáveis satisfação com a vida e esforço-recompensa com os fatores temporais (idade, anos de experiência na docência e anos de experiência na escola), embora seja necessário indagar sobre este aspecto. É importante destacar que o avanço no estudo dos fatores psicossociais que podem afetar a satisfação e a saúde ocupacional dos professores é essencial para a tomada de decisões políticas para a melhoria da educação no México. É necessário expandir esse tipo de pesquisa incluindo professores de diferentes níveis e contextos, de escolas públicas e privadas, a fim de obter informações mais precisas.

Palavras-chave: condições de emprego do professor, professor do ensino fundamental, fatores psicológicos, satisfação no trabalho.

Fecha Recepción: Febrero 2021

Fecha Aceptación: Agosto 2021

\section{Introducción}

En los últimos años, la globalización ha impulsado en el mundo una serie de cambios laborales, sociodemográficos, demográficos y políticos que han tenido importantes repercusiones en la salud de la población en general (Gil-Monte, 2009) y en la de los educadores en particular. De hecho, según diversas investigaciones (Aldrete, León, González, Hidalgo y Aranda, 2013; Alvites, 2019; Linares, 2014), los maestros son propicios a sufrir de estrés y otros padecimientos relacionados debido a una serie de circunstancias, como la alta carga administrativa, los grupos excesivamente numerosos, la cantidad de contenidos, la falta de tiempo, la carencia de herramientas, la falta de apoyo en casa, los bajos salarios, etc., lo cual provoca una situación difícil y peligrosa para la estabilidad emocional de dichos profesionales.

Expertos en seguridad y salud en el trabajo indican que las alteraciones que pueden afectar la salud psicológica de las personas son conocidas como riesgos psicosociales. El Comité Mixto de la Organización Internacional del Trabajo y la Organización Mundial de la Salud (OIT y OMS, 1984) define a los factores psicosociales en el trabajo de la siguiente manera:

Los factores psicosociales en el trabajo consisten en interacciones entre el trabajo, medio ambiente, la satisfacción en el trabajo y las condiciones de la organización, por una parte, y por la otra, las capacidades del trabajador, sus necesidades, su cultura y su situación personal fuera del trabajo, todo lo cual, a través de percepciones y experiencias, pueden influir en su salud y en el rendimiento y la satisfacción en el trabajo (p. 12). 


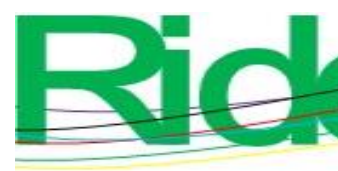

Revista Iberoamericana para la Investigación y el Desarrollo Educativo ISSN $2007-7467$

Tal como podemos suponer, la satisfacción con la vida podría ser un mecanismo personal para evaluar la relación entre el esfuerzo y la recompensa en el trabajo y, por otro lado, asociarse a una mejor perspectiva de salud laboral, entendida como el dominio y control de competencias instrumentales y sociales ligadas al puesto de trabajo.

Por todo lo anterior, el objetivo de este trabajo fue comparar en docentes de educación primaria las variables satisfacción con la vida, percepción de esfuerzo-recompensa y demandacontrol con los factores sexo, edad, antigüedad en la docencia, antigüedad en el centro de trabajo y atención a alumnos con necesidades educativas especiales (NEE).

\section{Método}

La presente fue una investigación no experimental, de tipo transeccional con alcance causal-comparativo (Creswell, 2009), para lo que se efectuó un procedimiento de muestreo no probabilístico por cuotas (McMillan y Schumacher, 2005). La recolección de información se realizó a través de la técnica de encuesta mediante la aplicación de diversos cuestionarios (para medir diferentes dimensiones de los factores psicosociales de riesgo) en hoja electrónica y leídos con un lector óptico. El diseño del método se sustentó en el paradigma positivista, que busca la objetividad y generalización de los resultados al controlar las variables de error, la aplicación de medidas y análisis estadístico (White y Sabarwal, 2014).

\section{Instrumentos}

Cuestionario de datos personales. Se utilizaron cuatro cuestionarios: el primero (con 13 reactivos) para recolectar información sobre sexo, edad, estado civil, cantidad de horas laborales en la escuela, tipo de contrato, nivel de formación, antigüedad en la docencia, antigüedad en el centro de trabajo, años de experiencia en el grado actual, estudiantes con NEE que atiende, entre otros.

Escala de satisfacción con la vida (SWLS). Se utilizó el instrumento de Diener et al. (1985), el cual consta de cinco reactivos que evalúan la satisfacción con la vida a través del juicio global que hacen las personas sobre esta (Padrós-Blázquez, Gutiérrez-Hernández y MedinaCalvillo, 2015). Los reactivos se respondieron en escala tipo Likert de cinco niveles, que iban desde totalmente en desacuerdo a totalmente de acuerdo. Las afirmaciones generales se enfocaron en qué tan satisfechos se encuentran con su vida. La puntuación total va de 5 (baja satisfacción) a 


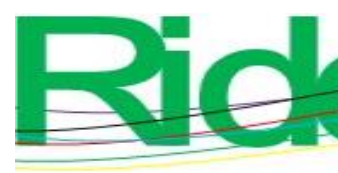

Revista Iberoamericana para la Investigación y el Desarrollo Educativo ISSN $2007-7467$

25 (alta satisfacción). La satisfacción global con la vida alude al componente cognitivo de bienestar, que explicó $43 \%$ de la varianza con un alfa de Cronbach de .80 (Vera, 2001).

Factores del modelo de desequilibrio esfuerzo-recompensa. Se utilizó la versión venezolana Cuestionario de estrés laboral (desbalance-esfuerzo/recompensa), validado por Díaz y Feldman (2010), el cual consiste en 22 reactivos en escala tipo Likert que van de totalmente en desacuerdo a totalmente de acuerdo (15 inclinados a un pico negativo y 7 hacia un pico positivo). Estos miden tres subdimensiones: recompensas, esfuerzo e implicaciones. Los primeros 6 ítems corresponden al factor esfuerzo, los siguientes 10 al factor recompensa y los últimos 6 al factor implicaciones.

Como consistencia interna, este instrumento cuenta con valores superiores a .80 en los análisis de alfa de Cronbach para las dimensiones recompensa y esfuerzo, mientras que para la dimensión implicación cuenta con .57 (Díaz y Feldman, 2010). No obstante, en estudios realizados en países europeos la fiabilidad de esta dimensión oscila entre .64 y valores superiores a .80 (Siegrist et al., 2004).

Factores del modelo demanda-control. El General Health Questionnaire (GQH) fue diseñado en 1979 por David Goldberg. En 1988 elaboró una versión de este, pero con 12 reactivos (GHQ-12) que evalúan la salud autopercibida y el estado de bienestar general, con énfasis en el estado emocional (Solís-Cámara, Meda, Moreno y Juárez, 2016).

Se utilizó el Cuestionario de salud general de Goldberg de 12 ítems (GHQ-12) en la versión cubana propuesta por García-Viniegras (1999). Se compone de 12 reactivos de opción múltiple con cuatro posibles respuestas cada uno: 6 de ellos se inclinan a un pico positivo (cuyas respuestas son más que lo habitual, igual que lo habitual, menos que lo habitual, mucho menos que lo habitual), mientras que los otros 6 a uno negativo (sus respuestas son no, en absoluto, no más que lo habitual, bastante más que lo habitual, mucho más).

Asimismo, maneja dos subdimensiones: bienestar psicológico (BP) y funcionamiento social y afrontamiento (FSA). La primera se define como un constructo que expresa el sentir positivo y el pensamiento constructivo del ser humano acerca de sí mismo, mientras que la segunda se refiere a la autoevaluación de la propia percepción de la capacidad para atender asuntos personales, afrontar preocupaciones, decidir y atender asuntos de la vida.

Se realizó un análisis factorial confirmatorio basado en el modelo de ecuaciones estructurales, respetando la estructura previamente planteada por los autores de cada escala para los doce reactivos. Los análisis mostraron una nueva estructura más apropiada para esta población, donde se extrajeron los reactivos 3, 4, 5, 7, 9 y 11 por no mostrar un apropiado ajuste. La estructura 

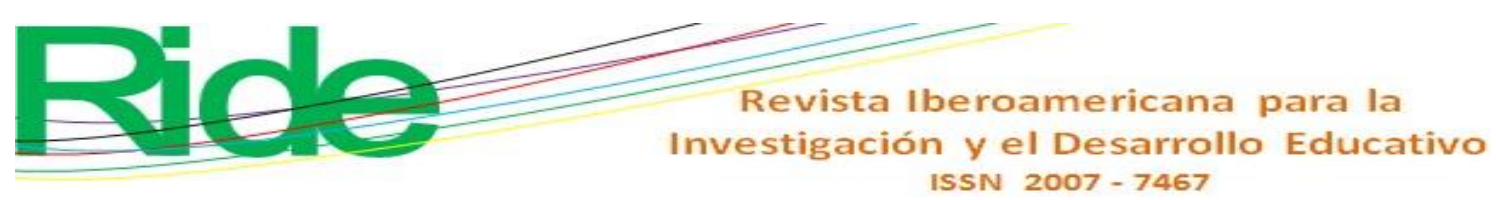

resultante se mantuvo con dos dimensiones: bienestar psicológico con tres reactivos $(1,2$ y 12$)$ y funcionamiento social y afrontamiento con tres reactivos (6, 8 y 11). Esta nueva estructura cuenta con los siguientes índices de bondad de ajuste, donde se obtuvo un CMIN/DF con valor de 2.52, CFI con valor de .944, RMSEA con valor de .06 y un SRMR de .04, los cuales son considerados como parámetros con un ajuste apropiado para la estructura propuesta (Gaskin, 2016).

\section{Participantes}

En la presente investigación participaron 185 docentes pertenecientes a 42 primarias del municipio de Cajeme, Sonora: 130 (70.27 \%) del turno matutino y 55 (29.72 \%) del vespertino. Para la elección de escuelas participantes se seleccionaron aquellas correspondientes al subsistema público (se excluyeron las de tipo multigrado y comunitarias), del turno matutino o vespertino, y ubicadas en colonias que cuentan con un alto número de reportes de violencia en las diferentes categorías: violencia intrafamiliar, omisión de cuidados, negligencia, abuso físico, psicológico, sexual, drogadicción e incumplimiento de obligaciones, esto de acuerdo con el Banco Estatal de Datos e Información sobre casos de Violencia contra las Mujeres (BAESVIM). Las escuelas fueron seleccionadas aleatoriamente y se aplicó el instrumento a los profesores de quinto y sexto grados de la institución presentes al momento de la evaluación.

Del total de docentes participantes, 88 (47.6 \%) fueron hombres y 97 (52.4\%) mujeres. Los rangos de edades fueron 20 (10.8\%) entre 18 y 25 años, 47 (25.4\%) entre 26 y 30 años, 43 (23.2 \%) entre 31 y 40 años, 49 (26.5\%) entre 41 y 50 años, y 25 (14.1\%) mayores de 50 años.

En cuanto al estado civil, participaron 56 (30.3\%) personas solteras, 103 (55.7\%) casadas, 16 (8.6.\%) en unión libre y 10 (5.4\%) separadas o divorciadas. Sobre el reactivo Cuenta con contrato indeterminado (plaza), 19 (10.3\%) respondieron negativamente (89.7\%) y 166 de manera afirmativa.

Sobre el nivel de formación, 100 (54.1\%) estudiaron una licenciatura por escuela normal, $37(20 \%)$ una licenciatura universitaria, $40(21.6 \%)$ cuentan con un posgrado en educación, dos $(1.1 \%)$ cuentan con un posgrado en otra especialidad y seis respondieron otra (3.2\%).

En lo referente a antigüedad en el servicio educativo, 48 (25.9\%) respondieron que cuentan con menos de 5 años de experiencia, 37 (20\%) de 6 a 10, 43 (23.2\%) de 11 a 20, 37 (20\%) de 21 a 30, y 20 (10.8 \%) cuentan con más de 30 años de experiencia. Sobre los años como docente en el centro educativo actual, 105 (56.8\%) respondieron que tienen menos de 5 años, 34 (18.4\%) tienen de 6 a 10 años, 33 (17.8 \%) tienen entre 11 y 20 años, y 13 (7\%) tienen de 21 a 30 años en 

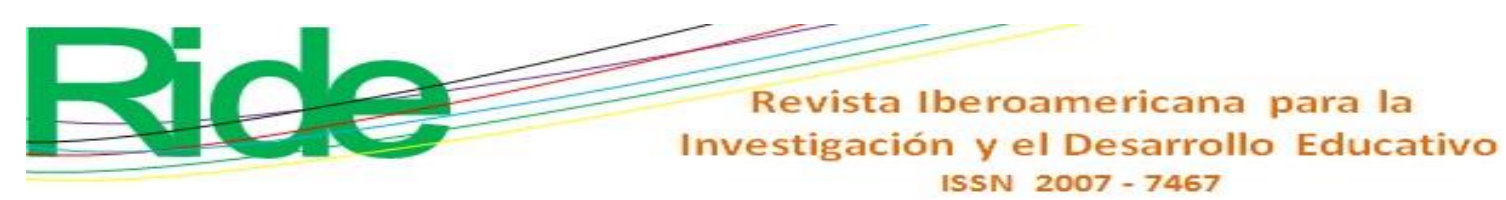

dicho centro. El reactivo Años de experiencia impartiendo el grado actual obtuvo los siguientes resultados: 127 (68.6\%) cuentan con menos de 5 años de experiencia, 43 (23.2 \%) de 6 a 10 años y $15(8.1 \%)$ de 11 a 20 años.

Por otra parte, al cuestionar sobre el Número aproximado de estudiantes con capacidades diferentes o con NEE que atiende, 41 (22.2\%) docentes respondieron que no atienden a ningún alumno con esas características, 75 (40.5\%) respondieron de 1 a 2 alumnos, 60 (32.4\%) de 3 a 5 alumnos, ocho de 6 a $10(4.3 \%)$ y solo uno (0.5\%) a más de 10.

\section{Procedimiento}

Para la puesta en marcha de esta investigación se seleccionaron escuelas pertenecientes al municipio de Cajeme, esto con ayuda de un listado proporcionado por la Secretaría de Educación y Cultura a través del Departamento de Salud y Seguridad Escolar. Una vez seleccionadas, se solicitaron los permisos correspondientes en dichas escuelas y se entregó un consentimiento informado donde se explicó a los docentes el objetivo, el procedimiento y la justificación del proyecto. Los instrumentos fueron aplicados de forma grupal a través de dos investigadores capacitados y estandarizados en el proceder frente a los docentes en el aula. El docente leía en un cuadernillo las preguntas y respondía en una hoja electrónica. El maestro de grupo siempre completo las preguntas en tiempos fuera de aula, todos firmaron un consentimiento informado e iniciaron sus respuestas.

Al terminar se revisaba que todas las respuestas fueran completadas, y durante el llenado se respondían dudas sobre las preguntas, aunque procurando que fuera el docente quien descubriera el significado de la pregunta sin sesgar sus respuestas. Después se procedió a aplicar el instrumento y a generar la base de datos utilizando el software SPSS, versión 22. Una vez capturada la información, se llevaron a cabo análisis descriptivos y de contraste de hipótesis para variables dicotómicas t de Student y análisis de varianza para las politómicas.

\section{Resultados}

\section{Análisis descriptivo}

Se efectuó un análisis descriptivo inicial para después realizar un contraste de hipótesis con los factores de atributo y las variables del estudio. Las diferentes medias por sexo y por grupos de edad resultan más altas en la variable demanda-control que en esfuerzo-recompensa. Por otro lado, se observa que en la medida en que aumenta el número de alumnos con NEE que se encuentran 


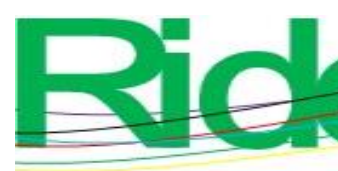

Revista Iberoamericana para la Investigación y el Desarrollo Educativo ISSN 2007 - 7467

bajo su responsabilidad, disminuye la media en las tres variables observadas: satisfacción con la vida, percepción esfuerzo-recompensa y demanda-control.

Los datos de punto de corte se determinaron mediante un análisis estadístico de cuartiles y deciles, y son los siguientes: se consideran sujetos con un nivel adecuado en el límite óptimo para la variable satisfacción con la vida, los que obtuvieron una media igual o mayor a 4.60, en el caso de la percepción esfuerzo-recompensa a quienes obtuvieron una media igual o mayor a 3.50, y para demanda-control los valores iguales o mayores a 3.58 (tabla 1).

Tabla 1. Resultados descriptivos para las variables de atributo docentes $(\mathrm{n}=185)$

\begin{tabular}{|c|c|c|c|c|c|c|c|}
\hline \multirow{2}{*}{\multicolumn{2}{|c|}{ Variables }} & \multicolumn{2}{|c|}{$\begin{array}{c}\text { Satisfacción con } \\
\text { la vida }\end{array}$} & \multicolumn{2}{|c|}{$\begin{array}{l}\text { Esfuerzo- } \\
\text { recompensa }\end{array}$} & \multicolumn{2}{|c|}{$\begin{array}{l}\text { Demanda- } \\
\text { control }\end{array}$} \\
\hline & & Media & D. E. & Media & D. E. & Media & D. E. \\
\hline \multirow{2}{*}{ Sexo } & Masculino & 4.06 & 0.77 & 3.2 & 0.46 & 3.41 & 0.36 \\
\hline & Femenino & 4.27 & 0.54 & 3.23 & 0.48 & 3.33 & 0.36 \\
\hline \multirow{5}{*}{ Edad } & 18 a 25 & 4.34 & 0.56 & 3.67 & 0.61 & 3.47 & 0.43 \\
\hline & 26 a 30 & 4.2 & 0.69 & 3.18 & 0.37 & 3.34 & 0.37 \\
\hline & 31 a 40 & 4.13 & 0.75 & 3.15 & 0.54 & 3.38 & 0.28 \\
\hline & 41 a 50 & 4.2 & 0.65 & 3.16 & 0.37 & 3.35 & 0.38 \\
\hline & Mayor de 50 & 4 & 0.59 & 3.15 & 0.41 & 3.36 & 0.36 \\
\hline \multirow{5}{*}{$\begin{array}{l}\text { Años de } \\
\text { experiencia } \\
\text { en la } \\
\text { docencia }\end{array}$} & Menos de 5 & 4.25 & 0.7 & 3.44 & 0.56 & 3.39 & 0.41 \\
\hline & de 6 a 10 & 4.17 & 0.52 & 3.17 & 0.32 & 3.36 & 0.37 \\
\hline & de 11 a 20 & 4.17 & 0.83 & 3.06 & 0.46 & 3.4 & 0.31 \\
\hline & de 21 a 30 & 4.09 & 0.6 & 3.15 & 0.4 & 3.32 & 0.35 \\
\hline & más de 30 & 4.12 & 0.57 & 3.2 & 0.45 & 3.34 & 0.34 \\
\hline \multirow{4}{*}{$\begin{array}{l}\text { Años como } \\
\text { docente en } \\
\text { esta escuela }\end{array}$} & Menos de 5 & 4.24 & 0.61 & 3.31 & 0.51 & 3.4 & 0.34 \\
\hline & de 6 a 10 & 3.94 & 0.81 & 3.11 & 0.37 & 3.34 & 0.41 \\
\hline & de 11 a 20 & 4.21 & 0.59 & 3.12 & 0.42 & 3.34 & 0.39 \\
\hline & de 21 a 30 & 4.06 & 0.77 & 2.97 & 0.32 & 3.24 & 0.23 \\
\hline \multirow{4}{*}{$\begin{array}{l}\text { Número de } \\
\text { alumnos con } \\
\text { NEE que } \\
\text { atiende }\end{array}$} & ninguno & 4.34 & 0.53 & 3.31 & 0.47 & 3.47 & 0.24 \\
\hline & 1 o 2 & 4.14 & 0.78 & 3.22 & 0.5 & 3.38 & 0.37 \\
\hline & de 3 a 5 & 4.1 & 0.6 & 3.14 & 0.44 & 3.29 & 0.42 \\
\hline & de 6 a 10 & 4 & 0.51 & 3.25 & 0.42 & 3.35 & 0.21 \\
\hline
\end{tabular}

Fuente: Elaboración propia

Por otra parte, se consideran sujetos estables con oportunidades de mejora a quienes obtuvieron valores medios: en la variable satisfacción con la vida entre 3.41 y 4.59, en la variable percepción esfuerzo-recompensa a aquellos con una media entre 2.69 y 3.49 y, por último, en la variable demanda-control a aquellos con valores entre 3.01 y 3.57 . 
Tabla 2. Resultados del análisis de varianza para los factores politómicos de atributo

\begin{tabular}{|c|c|c|c|c|c|c|c|}
\hline \multirow{2}{*}{\multicolumn{2}{|c|}{ Variables }} & \multicolumn{3}{|c|}{ Esfuerzo-recompensa } & & & \multirow{2}{*}{$\begin{array}{c}\begin{array}{c}\text { Tamaño del } \\
\text { efecto }\end{array} \\
(f) \\
\end{array}$} \\
\hline & & $\mathrm{n}$ & Media & D. E. & $\mathrm{F}$ & Sig. & \\
\hline \multirow{5}{*}{ Edad } & 18 a 25 & 20 & 3.67 & 0.61 & \multirow{5}{*}{5.62} & \multirow{5}{*}{0.000} & \\
\hline & 26 a 30 & 47 & 3.18 & 0.37 & & & \\
\hline & 31 a 40 & 43 & 3.15 & 0.54 & & & .33 \\
\hline & 41 a 50 & 49 & 3.16 & 0.37 & & & \\
\hline & Mayor de 50 & 26 & 3.15 & 0.41 & & & \\
\hline \multirow{5}{*}{$\begin{array}{l}\text { Años de } \\
\text { experiencia } \\
\text { docente }\end{array}$} & Menos de 5 & 48 & 3.44 & 0.56 & \multirow{5}{*}{4.43} & \multirow{5}{*}{0.002} & \\
\hline & de 6 a 10 & 37 & 3.17 & 0.32 & & & \\
\hline & de 11 a 20 & 43 & 3.06 & 0.46 & & & .30 \\
\hline & de 21 a 30 & 37 & 3.15 & 0.4 & & & \\
\hline & más de 30 & 20 & 3.2 & 0.45 & & & \\
\hline \multirow{4}{*}{$\begin{array}{l}\text { Años de } \\
\text { experiencia } \\
\text { en la escuela }\end{array}$} & Menos de 5 & 105 & 3.31 & 0.51 & & & \\
\hline & de 6 a 10 & 34 & 3.11 & 0.37 & 3.65 & 0.014 & .24 \\
\hline & de 11 a 20 & 33 & 3.12 & 0.42 & & & \\
\hline & de 21 a 30 & 13 & 2.97 & 0.32 & & & \\
\hline
\end{tabular}

Fuente: Elaboración propia

Se obtuvieron los deciles para cada una de las variables. La variable demanda-control obtuvo en el primer decil el valor de 3.00, lo cual coloca a 22 personas (11.9\%) debajo de este valor, considerado como el nivel más bajo y, por tanto, no adecuado en la variable demandacontrol, lo cual significa que se encuentran en riesgo de un problema de salud mental. Por otra parte, en la variable percepción esfuerzo-recompensa 21 individuos (11.4\%) presentaron niveles por debajo del primer decil con un valor de $2.68 \mathrm{y}$, por tanto, desequilibrado, perciben que el esfuerzo que realizan es mayor a las recompensas que reciben por ello en su trabajo. Por último, el valor del primer decil fue de 3.40 para 22 personas (11.9\%), que representa los niveles más bajos en la escala de satisfacción con la vida.

En el caso de la variable demanda-control, no parece haber un cambio significativo con el paso del tiempo, aunque sí se observa una disminución mínima a diferencia de la variable percepción esfuerzo-recompensa, en la que el tiempo sí tiene un efecto: de acuerdo con los resultados de este estudio, con el paso de los años la percepción de recibir lo merecido por el esfuerzo realizado va disminuyendo significativamente o, dicho de otra manera, con el tiempo se afianza la creencia de que se requieren más recompensas por el esfuerzo realizado.

La variable demanda-control no genera diferencias significativas debido a que el docente establece una relación personal entre el compromiso de enseñar y que los infantes aprendan y la propia capacidad de llevarlo a cabo. De esta forma se genera un sentimiento de desamparo al 


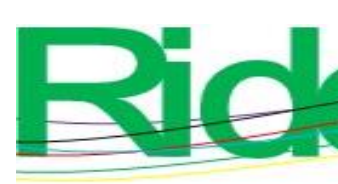

Revista Iberoamericana para la Investigación y el Desarrollo Educativo ISSN $2007-7467$

percibir poco o nulo apoyo de sus autoridades, padres de familia o compañeros, por lo que se crea un compromiso intrínseco por su labor y, por tanto, de control en su trabajo.

Continuando con el análisis, se observa que el factor Número de alumnos con NEE que atiende no presenta diferencias significativas en ninguna de las variables estudiadas: satisfacción con la vida, percepción esfuerzo-recompensa y demanda-control.

Finalmente, se llevó a cabo un contraste de hipótesis para variables dicotómicas mediante la $\mathrm{t}$ de Student comparando hombres contra mujeres, y se encontró que las mujeres tienen una media mayor de satisfacción con la vida que los hombres, con un valor de $t$ de 2.12 y una significancia de .03 .

\section{Discusión}

El análisis descriptivo permitió comparar diferentes factores con las tres variables estudiadas y, con esto, se evidencia que el número de alumnos con NEE que se atienden influye en la satisfacción con la vida, percepción esfuerzo-recompensa y demanda-control: a medida en que aumenta el número de alumnos atendidos con dichas características disminuye la media en las tres variables. En relación con lo anterior, Amezcua-Sandoval, Preciado-Serrano, Pando-Moreno y Salazar-Estrada (2011) encontraron que de la muestra de docentes de educación especial incluidos en su investigación, $11.1 \%$ presentaron sintomatología depresiva, cifra superior a la reportada para la población adulta en México en 2005.

Esta falta de habilidades y competencias para la inclusión no son privativas de la educación básica, sino que permean todos los niveles educativos en los cuales dicho grupo se encuentra segregado, excluido y maltratado (García, 2019; Pérez, 2016; Zárate, Díaz y Ortiz, 2017). Aun cuando el diagnóstico esté basado en creencias o suposiciones del docente sobre la problemática del alumno, se puede observar por las medias de satisfacción que el grado se encuentra entre medio-alto, lo cual coincide con los hallazgos de Anaya y Suárez (2010) y Linares y Gutiérrez (2010).

Por otra parte, encontramos que el factor temporal también influye en las variables estudiadas: tanto el reactivo Años de experiencia en la docencia y Años como docente en la escuela presentan una tendencia a la baja ante la variable demanda-control; así pues, mientras menos experiencia o edad se tiene, parece presentarse mayor satisfacción con la relación demanda-control en el trabajo, o bien al ser un instrumento que mide el nivel de salud autopercibida; entonces, concluimos que a menor edad mayor percepción de buena salud. Cabe destacar que el instrumento 


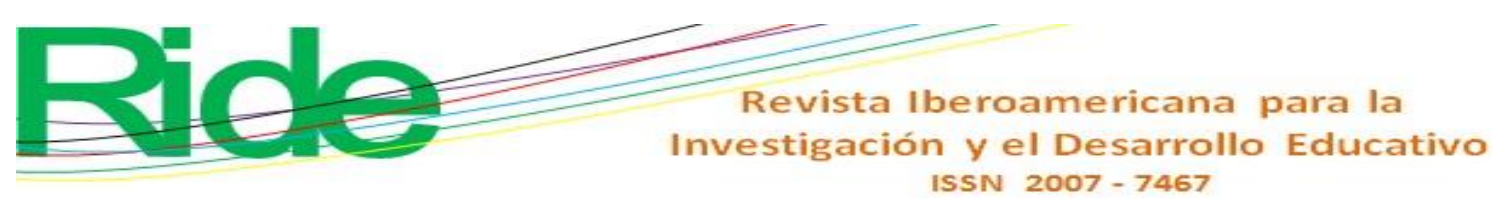

de Goldberg se enfoca en la salud mental. Lo anterior también ocurre con la variable satisfacción con la vida: mientras menos edad o años de experiencia se tienen se observa un mayor nivel de satisfacción. Al respecto, Clark, Oswald y Warr (1996) y Womack-Wynne et al. (2011) expresan que los trabajadores de recién ingreso sienten mayor satisfacción al estar en una situación nueva y por el simple hecho de pertenecer al mercado laboral del cual no conocen lo suficiente para llevar a cabo una evaluación objetiva de su trabajo. Por ejemplo, entre la población activa de un condado sueco un alto nivel de agotamiento fue más común entre los trabajadores de edad avanzada (Ahola, Honkonen, Virtanen, Aromaa y Lönnqvist, 2008).

Al respecto, Carrillo-García, Solano-Ruiz, Martínez-Roche y Gómez-García (2013) mencionan que no hay unanimidad en los estudios sobre la edad y su influencia en diferentes aspectos del ámbito laboral o personal. Por un lado, se señala que la satisfacción laboral aumenta con la edad (Alonso, 2008; Malander, 2016), mientras que otros estudios mencionan que a mayor edad menor satisfacción (Hermosa, 2006; Llorent y Ruiz-Calzado, 2016).

Sobre la variable demanda-control se encontraron muy pocas variaciones, lo cual coincide con los hallazgos de Villa, Zuluaga-Arboleda y Restrepo-Roldán (2013), quienes sugieren con base en la poca variación en los resultados de un estudio llevado a cabo con pacientes de un hospital en Medellín, Colombia, que la percepción de bienestar o malestar que arroja el instrumento GHQ12 se relaciona principalmente con factores intrínsecos más que con factores extrínsecos.

Es importante hacer notar que la relación demanda-control asociada a la eficacia del docente para cumplir con la labor educativa se vincula con la satisfacción laboral (Viel-Ruma, Houchins, Jolivette y Benson, 2010) y resulta en un antecedente de problemáticas de burnout en docentes (Aldrete, Aranda, Valencia y Salazar, 2011; Hermosa, 2006).

Respecto a la satisfacción con la vida, según algunos estudios, siempre resulta mayor para hombres que para mujeres (Expósito, Agost-Felip y Soto, 2015; Moreta-Herrera, López-Calle, Gordón-Villalba, Ortiz-Ochoa y Gaibor-González, 2018); sin embargo, en nuestros datos sucede lo contrario, por lo que debemos tener en cuenta que se trata de un colectivo laboral que históricamente ha sido feminizado, y es posible que las mujeres en este contexto femenino se perciban más satisfechas. En cuanto a las limitaciones del estudio, debemos mencionar inicialmente los sesgos y errores que genera un estudio retrospectivo con muestras no aleatorias; en segundo plano, la forma en la cual pueden afectar la validez externa el que la población solo sea de un municipio. Sin embargo, resulta importante destacar que avanzar en el estudio de los factores psicosociales que pueden afectar la satisfacción y salud laboral del magisterio es 

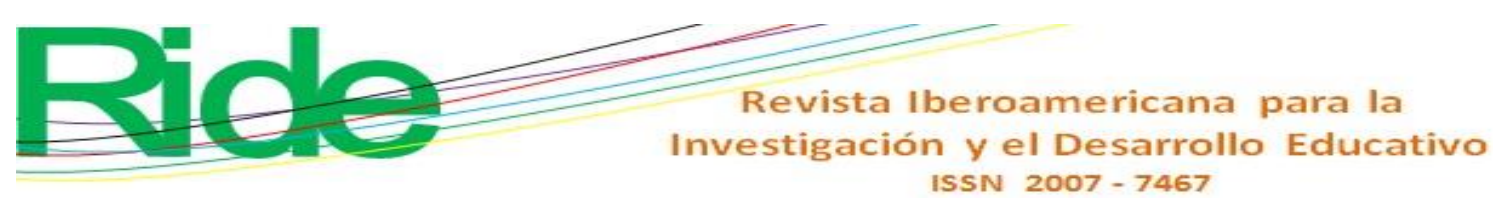

profesión docente, la forma en que el equilibrio esfuerzo-recompensa y la salud mental pueden impactar su comportamiento social en la escuela y el aula, y desencadenar emociones negativas que promuevan conductas antisociales en los alumnos y constituir un obstáculo para lograr una cultura de paz.

\section{Referencias}

Ahola, K., Honkonen, T., Virtanen, M., Aromaa, A. and Lönnqvist, J. (2008). Burnout in relation to age in the adult working population. Journal of Occupational Health, 50(4), 362-365. Doi: https://doi.org/10.1539/joh.M8002

Aldrete, M. G., Aranda, C., Valencia, S. y Salazar, J. G. (2011). Satisfacción laboral y síndrome de burnout en docentes de secundaria. Revista Educación y Desarrollo, 17, 15-22. Recuperado de http://www.cucs.udg.mx/revistas/edu_desarrollo/anterioresdetalle.php?n=17

Aldrete, M., León, S., González, R., Hidalgo, G. y Aranda, C. (2013). Estrés y factores psicosociales laborales en profesoras del preescolar de la zona metropolitana de Guadalajara. Revista de Educación y Desarrollo, 28, 69-75. Recuperado de https://www.cucs.udg.mx/revistas/edu_desarrollo/anteriores/28/028_Aldrete.pdf

Alonso, P. (2008). Estudio comparativo de la satisfacción laboral en el personal de administración. Revista de Psicología del Trabajo y de las Organizaciones, 24(1), 25-40. Recuperado de http://scielo.isciii.es/scielo.php?script=sci_arttext\&pid=S1576$59622008000100002 \& \operatorname{lng}=$ es\&tlng=es

Alvites, C. (2019). Estrés docente y factores psicosociales en docentes de Latinoamérica, Norteamérica y Europa. Propósitos y Representaciones, 7(3), 141-178. Doi: http://dx.doi.org/10.20511/pyr2019.v7n3.393

Amezcua-Sandoval, M. T., Preciado-Serrano, L., Pando-Moreno, M. y Salazar-Estrada, J. G. (2011). Factores psicosociales y sintomatología depresiva en profesionales docentes que trabajan con alumnos especiales. Educación y Desarrollo, 19(25), 67-72. Recuperado de https://nanopdf.com/download/factores-psicosociales-y-sintomatolog-a-depresiva-enprofesionales-docentes-que_pdf

Anaya, D. y Suárez, J. M. (2010). Evaluación de la satisfacción laboral de profesorado y aportaciones a su mejora en orden a la calidad de la educación. Revista Española de Orientación y Psicopedagogía, 21(2), 283-294. 

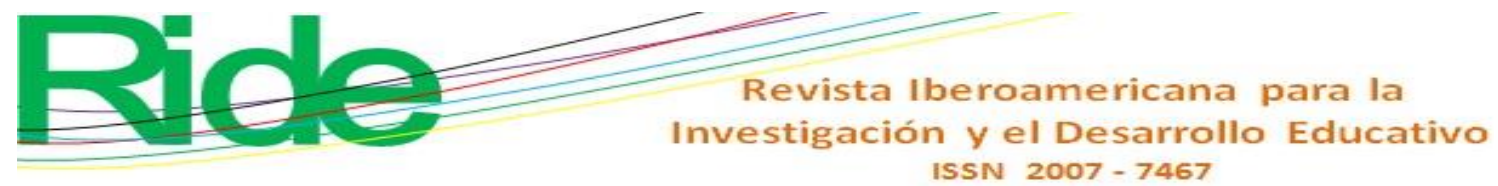

García-Viniegras, C. R. (1999). Manual para la utilización del cuestionario de salud general de Goldberg: adaptación cubana. Revista Cubana de Medicina General Integral, 15(1), 8897. Recuperado de http://scielo.sld.cu/pdf/mgi/v15n1/mgi10199.pdf

Gaskin, J. (2016). Stat Wiki. Retrieved from http://statwiki.kolobkreations.com/index.php?title=Main_Page

Gil-Monte, P. R. (2009). Algunas razones para considerar los riesgos psicosociales en el trabajo y sus consecuencias en la salud pública. Revista Española de Salud Pública, 83(2), 169-173. Recuperado de http://scielo.isciii.es/pdf/resp/v83n2/editorial3.pdf

Gómez-Ortiz, V. y Moreno, L. (2010). Factores psicosociales del trabajo (demanda-control y desbalance esfuerzo-recompensa), salud mental y tensión arterial: un estudio con maestros escolares en Bogotá, Colombia. Universitas Psychologica, 9(2), 393-407. Recuperado de http://pepsic.bvsalud.org/pdf/up/v9n2/v9n2a08.pdf.

Halpert, M. A. (2011). Factors affecting teacher satisfaction in an urban school district. Proquest Llc. Retrieved from http://search.proquest.com/docview/861731227

Hermosa, A. M. (2006). Satisfacción laboral y síndrome de "burnout" en profesores de educación primaria y secundaria. Revista Colombiana de Psicología, 15(1), 81-89. Recuperado de https://www.redalyc.org/articulo.oa?id=80401509

Jiménez, A., Jara, M. y Miransa, E. (2012). Burnout, apoyo social y satisfacción laboral en docentes. Psicologia Escolar e Educacional, 16(1), 125-134. Doi: https://doi.org/10.1590/S1413-85572012000100013

Karasek, R. (1979). Job demands, job decision latitude, and mental strain: implications for job redesign. Administrative Science Quaraterly, 24(2), 285-308. Doi: https://doi.org/10.2307/2392498

Linares, O. L. (2014). Influencia del estrés, la ansiedad y el apoyo en el trabajo sobre el nivel de satisfacción laboral, malestar físico y ausentismo en profesores (tesis de doctorado), México: Universidad Nacional Autónoma de México. Recuperado de https://ru.dgb.unam.mx/handle/DGB_UNAM/TES01000707608

Linares, O. y Gutiérrez, R. (2010). Satisfacción laboral y percepción de salud mental en profesores. Revista Mexicana de Investigación en Psicología, 2(1), 31-36.

Llorent, V. J. y Ruiz-Calzado, I. (2016). El burnout y las variables sociodemográficas en los profesionales de la educación que trabajan con personas con discapacidad en Córdoba (España). Ciência \& Saúde Coletiva, 21(10), 3287-3295. Doi: https://doi.org/10.1590/1413-812320152110.00732015 


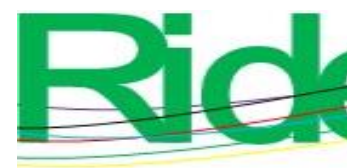

Revista Iberoamericana para la Investigación y el Desarrollo Educativo ISSN $2007-7467$

Llull, D. S., Cerdà, M. X. M. y Brage, L. B. (2015). Malestar social y malestar docente: una investigación sobre el síndrome de desgaste profesional burnout y su incidencia socioeducativa. Aula: Revista de Pedagogía de la Universidad de Salamanca, 21(1), 245257. Doi: http://dx.doi.org/10.14201/aula201521245257

Malander, N. M. (2016). Síndrome de burnout y satisfacción laboral en docentes de nivel secundario. Ciencia \& Trabajo, 18(57), 177-182. Doi: https://dx.doi.org/10.4067/S071824492016000300177

McMillan, J. y Schumacher, S. (2005). Investigación educativa, una introducción conceptual (5. ${ }^{\mathrm{a}}$ ed.). Madrid: Pearson educación.

Moreta-Herrera, R., López-Calle, C., Gordón-Villalba, P., Ortiz-Ochoa, W. y Gaibor-González, I. (2018). Satisfacción con la vida, bienestar psicológico y social como predictores de la salud mental en ecuatorianos. Actualidades en Psicología, 32(124), 112-126. Doi: http://dx.doi.org/10.15517/ap.v32i124.31989

Organización Internacional del Trabajo y Organización Mundial de la Salud (1984). Factores psicosociales en el trabajo: naturaleza, incidencia y prevención. Recuperado de http://www.factorespsicosociales.com/wp-content/uploads/2019/02/FPS-OIT-OMS.pdf

Padrós-Blázquez, F., Gutiérrez-Hernández, C. Y. y Medina-Calvillo, M. A. (2015). Propiedades psicométricas de la escala de satisfacción con la vida (SWLS) de Diener en población de Michoacán (México). Avances en Psicología Latinoamericana, 33(2), 223-232. Recuperado de http://www.redalyc.org/articulo.oa?id=79938616004

Pérez, J. (2016). La inclusión de las personas con discapacidad en la educación superior en México. Sinéctica, Revista Electrónica de Educación, 46, 1-15. Recuperado de http://www.redalyc.org/articulo.oa?id=99843455011

Siegrist, J. (1996). Adverse health effects of high effort /low reward conditions. Journal of Occupational Health Psychology, 1(1), 27-41. Doi: https://doi.org/10.1037//10768998.1.1.27

Siegrist, J., Starke, D., Chandola, T., Godin, I., Marmot, M., Niedhammer, I. and Peter, R. (2004). The measurement of effort-reward imbalance at work: European comparisons. Social Science \& Medicine, 58, 1483-1499. Doi: https://doi.org/10.1016/S0277-9536(03)003514

Solís-Cámara, P., Meda, L. R., Moreno, J. B. y Juárez, R. P. (2016). Estructura factorial del cuestionario de salud general GHQ-12 en población general de México. Salud \& Sociedad, 7(1) 62-76. Doi: https://doi.org/10.22199/S07187475.2016.0001.00004 


\begin{tabular}{|c|c|}
\hline Rol de Contribución & Autor (es) \\
\hline Conceptualización & JOSE ANGEL VERA NORIEGA \\
\hline Metodología & JOSE ANGEL VERA NORIEGA \\
\hline Software & NO APLICA \\
\hline Validación & JOSE ANGEL VERA NORIEGA \\
\hline Análisis Formal & $\begin{array}{l}\text { JAZMÍN VALDEZ TAM (IGUAL) } \\
\text { ERIKA SELENE CONTRERAS GRIJALVA (IGUAL) } \\
\text { SILVIA SELENE CASTILLO VELASCO (IGUAL) }\end{array}$ \\
\hline Investigación & $\begin{array}{l}\text { JOSE ANGEL VERA NORIEGA (PRINICPAL) } \\
\text { JAZMÍN VALDEZ TAM (IGUAL) } \\
\text { ERIKA SELENE CONTRERAS GRIJALVA (IGUAL) } \\
\text { SILVIA SELENE CASTILLO VELASCO (IGUAL) }\end{array}$ \\
\hline Recursos & JOSE ANGEL VERA NORIEGA \\
\hline Curación de datos & $\begin{array}{l}\text { JOSE ANGEL VERA NORIEGA (PRINICPAL) } \\
\text { JAZMÍN VALDEZ TAM (IGUAL) } \\
\text { ERIKA SELENE CONTRERAS GRIJALVA (IGUAL) } \\
\text { SILVIA SELENE CASTILLO VELASCO (IGUAL) }\end{array}$ \\
\hline $\begin{array}{l}\text { Escritura - Preparación del } \\
\text { borrador original }\end{array}$ & $\begin{array}{l}\text { JOSE ANGEL VERA NORIEGA (PRINICPAL) } \\
\text { JAZMÍN VALDEZ TAM (IGUAL) } \\
\text { ERIKA SELENE CONTRERAS GRIJALVA (IGUAL) } \\
\text { SILVIA SELENE CASTILLO VELASCO (IGUAL) }\end{array}$ \\
\hline $\begin{array}{l}\text { Escritura - Revisión y } \\
\text { edición }\end{array}$ & $\begin{array}{l}\text { JOSE ANGEL VERA NORIEGA (PRINICPAL) } \\
\text { JAZMÍN VALDEZ TAM (IGUAL) } \\
\text { ERIKA SELENE CONTRERAS GRIJALVA (IGUAL) } \\
\text { SILVIA SELENE CASTILLO VELASCO (IGUAL) }\end{array}$ \\
\hline Visualización & $\begin{array}{l}\text { JOSE ANGEL VERA NORIEGA (PRINICPAL) } \\
\text { JAZMÍN VALDEZ TAM (IGUAL) } \\
\text { ERIKA SELENE CONTRERAS GRIJALVA (IGUAL) } \\
\text { SILVIA SELENE CASTILLO VELASCO (IGUAL) }\end{array}$ \\
\hline Supervisión & JOSE ANGEL VERA NORIEGA \\
\hline Administración de Proyectos & JOSE ANGEL VERA NORIEGA \\
\hline Adquisición de fondos & JOSE ANGEL VERA NORIEGA \\
\hline
\end{tabular}

